\title{
Recurrent laryngeal nerve paralysis in patients receiving vincristine and vinblastine
}

\author{
J A WHITTAKER, I P GRIFFITH
}

British Medical fournal, 1977, 1, 1251-1252

\section{Case 2}

A 27 -year-old dentist presented in January 1974 with a one-month history of pain and swelling in the left clavicular region and left axilla. Examination showed multiple non-painful nodes about $3 \mathrm{~cm}$ in diameter in the left axilla and left side of the neck. Node biopsy showed nodular sclerosing Hodgkin's disease. Other investigations, including chest radiography, lymphangiography, and bone marrow trephine were normal. Staging laparotomy and splenectomy in February 1974 showed a macroscopically normal liver and spleen. The coeliac nodes were enlarged but no other intra-abdominal lymphadenopathy was apparent. Histologically the spleen and coeliac lymph nodes were infiltrated with Hodgkin's disease but the liver was unaffected. Treatment started in April 1974: intravenous vincristine $2.5 \mathrm{mg}$ and mustine $10.8 \mathrm{mg}$ were given and the doses were repeated after seven days; oral procarbazine $200 \mathrm{mg} /$ day was given for 10 days and oral prednisone $45 \mathrm{mg} /$ day for 14 days. The doses of vincristine and mustine were repeated after 14 days. A second course of treatment was given in May 1976. When the third course was given in June the patient complained of intermittent hoarseness. There were no abnormal physical signs, but in July, when the hoarseness had worsened, indirect laryngoscopy showed a left recurrent laryngeal nerve palsy. Vincristine was omitted from further treatment courses. In August the patient's voice was much improved and he had not had hoarseness for a week. Subsequent indirect laryngoscopy showed normal vocal cords, which were freely mobile. Treatment was completed in September 1974, and the patient subsequently remained well.

\section{Case 3}

A 27-year-old man first presented in March 1975 with a mass in his left axilla, which he had had for two years. The mass had suddenly become larger and examination showed two other lumps in his neck. Biopsy of an axillary lymph node showed Hodgkin's disease of mixed cell type. Marrow trephine and lymphangiogram were normal. Laparotomy and splenectomy showed macroscopically normal liver and spleen but histological examination showed that nodes from the lesser curve of the stomach and the spleen were infiltrated with Hodgkin's disease of mixed cell type. Treatment was started in April 1975 with intravenous vincristine $2.8 \mathrm{mg}$ and mustine $12 \mathrm{mg}$. These doses were repeated after seven days. Procarbazine $200 \mathrm{mg} /$ day was given for 10 days and Prednisone $50 \mathrm{mg} /$ day for 14 days. Second and third courses were given in May and June. In June he complained of a persistent dry cough, which he had had for two weeks. There were no abnormal physical signs, his temperature was normal, and his lung fields were clear. A chest radiograph was normal. Two weeks later indirect laryngoscopy was performed because his cough had become worse. This showed left vocal cord paralysis. Vincristine treatment was discontinued and bleomycin $30 \mathrm{mg}$ intravenously was substitued. Within a month his cough and hoarseness had disappeared. Fourth and fifth courses were given in August and September, but he refused the final course of treatment. Repeat indirect laryngoscopy showed normal vocal cords, which were freely mobile. The patient has since remained well and free of disease.

\section{Discussion}

As well as their established role in the treatment of acute lymphoblastic leukaemia and the lymphomas, vincristine and the closely related vinca alkaloid vinblastine have a place in the treatment of various other tumours. Both drugs are neurotoxic, although vincristine seems to cause more adverse effects than vinblastine, especially in adults, in whom the
University Hospital of Wales, and Welsh National School of Medicine, Cardiff CF4 4XW

J A WHITTAKER, MD, MRCP, senior lecturer and consultant haematologist throat surgeon 
incidence of side effects is three times that in children. ${ }^{4}$ Loss of tendon reflexes is the first sign of toxicity, and this is followed by paraesthaesiae, sometimes with distal sensory loss. ${ }^{5}$ If the drug is not stopped distal muscle weakness and wasting occur. Vincristine may damage the autonomic nervous system, producing constipation, a common side effect, or occasionally urinary retention. The degree of toxicity is related to the dose, and the adverse effects usually disappear when the drug is withdrawn.

References to specific laryngeal dysfunction in patients receiving vincristine appear in early reports on the drug. Bohannon et al reported that five out of 35 patients receiving vincristine for late stage resistant leukaemia or lymphoma developed hoarseness. ${ }^{3}$ In that study, however, the doses were higher than are currently acceptable, and with lower doses the danger of recurrent laryngeal nerve damage may have been forgotten. More recently ophthalmoplegia and hoarseness have been mentioned as a complication of vincristine treatment, ${ }^{\circ}$ and recurrent laryngeal nerve damage has been reported in two patients receiving treatment with vinblastine.?

When a patient with a known lymphoma presents with hoarseness it is tempting to assume that this is related to pressura from mediastinal lymphadenopathy. Our experience suggests that this is far less likely than recurrent laryngeal nerve palsy secondary to vinca alkaloid treatment. Two of our patients (cases 2 and 3 ) had shown mediastinal lymphadenopathy at presentation, although it was not visible on chest radiographs when they developed hoarsencss. Furthermore, one patient (case 1) had a right vocal cord palsy. Three of the patients described previously ${ }^{6}$ had a left vocal cord paralysis but the state of the mediastinal lymph nodes was not mentioned.

Like others, ${ }^{7}$ we found that the paralysis in our three patients completely disappeared after the drug was withdrawn. Patients who develop hoarseness during treatment may be thought to have an infection, and consequently the hypopharynx and larynx may not always be examined properly. The knowledge that these drugs are neurotoxic should prompt a more careful laryngeal screening, so that vocal cord paralysis can be detected early and progression to bilateral paralysis, with the danger of respiratory distress, can be prevented.

\section{References}

1 Johnson, I S, et al, Cancer Reseurch, 1963, 23, 1390.

2 Casey, E B, et al, Brain, 1973, 96, 69.

3 Bohannon, R A, Miller, D G, and Diamond, H D, Cancer Research, 1963, 23, 613.

4 Whitelaw, D M, et al, Cancer Chemotherapy Reports, 1963, 30, 13.

5 Lancet, 1973, 1, 980.

6 Holland, J F, et al, Cancer Research, 1973, 33, 1258.

7 Brook, J, and Schreiber, W, Cancer Chemotherapy Reports, 1971, 55, 591.

\title{
Control of blood glucose during labour in diabetic women with combined glucose and low-dose insulin infusion
}

\author{
T E T WEST, C LOWY
}

immature infants as well as in infants of diabetic mothers) is much reduced by delivery after 38 weeks.

Control of the maternal blood glucose concentrations during

\section{Summary}

During 15 labours in diabetic women blood glucose concentrations were controlled with simultaneous infusion of insulin and glucose. The mean insulin infusion rate was between 1 and $2 U / h$. No infant showed evidence of neonatal hypoglycaemia. The procedure is simple to use and may be carried out in any labour ward.

\section{Introduction}

Since insulin treatment first became available the numbers of diabetic women becoming pregnant have increased dramatically. This is due to decreased mortality and increased fertility. Risks to the mother and fetus in diabetic pregnancies have been well established, and it is now established that careful control of blood glucose concentrations (particularly in the last trimester) usually results in the birth of a healthy, normal-weight infant at low risk of developing neonatal hypoglycaemia. The incidence of neonatal respiratory distress syndrome (commoner in

St Thomas's Hospital Medical School, London SE1 7EH

T E T WEST, MD, MRCP, lecturer in medicine

C LOWY, MSC, MRCP, senior lecturer in chemical pathology the use of shorter-acting insulins but give no clear-cut advice the provision of energy requirements. This is important because labour is often prolonged in diabetic mothers. Gastric emptying is delayed in pregnancy and is erratic during labour. This poses the twin problems of correct judgment of insulin dose and preparedness of the mother for general anaesthesia should this be needed-for example, for caesarean section.

We have overcome these problems by modifying the continuous low-dose intravenous insulin infusion technique developed for treating diabetic ketoacidosis ${ }^{1}$ : glucose and insulin are infused simultaneously. We report here the results of using this form of management in 15 labours.

\section{Patients and methods}

Fourteen patients were studied (see table), one of them (case 1) being treated twice during labour after two consecutive successful pregnancies. All except two of the patients were established diabetics receiving long-term insulin treatment. In case 2 the patient had given birth to a large-for-dates baby $(6000 \mathrm{~g})$ eight years previously and was first treated with insulin during the present pregnancy, and the other patient (case 8) first presented with diabetes at 15 weeks' gestation. All patients were closely monitored in the antenatal clinic by an obstetrician and a diabetologist. Blood glucose was carefully controlled. Hospital admission during the earlier stages of pregnancy was advised only for radical changes in insulin treatment or when the blood glucose concentration escaped satisfactory control.

Patients were admitted to hospital one to four weeks before the 